\title{
ALS ZUHOORER BEI EINEM CHINESISCHEN STRAFVERFAHREN
}

\author{
Von Harago SanjI*
}

\section{Einleitung}

Vom 9. bis 20. 12. 1974 hatte ich Gelegenheit, als Mitglied einer von der VR China zu einem Freundschaftsbesuch eingeladenen Gruppe von Vertretern der japanischen Anwaltschaft Peking, Suzhou und Shanghai zu besuchen. Davor hatte zum letzten Mal 1965 eine Gruppe japanischer Anwälte China besucht; als erste japanische Anwaltsgruppe seit der Kulturrevolution waren wir anläßlich des Inkrafttretens des chinesisch-japanischen Luftverkehrsabkommens von der chinesischen Regierung eingeladen worden. Unsere Gruppe - sieben Anwälte unter Leitung des Vorsitzenden des Tokyoter Anwaltsvereins, Kashiwagi Hiroshi, wurde in China von allen, mit denen wir es zu tun hatten, vor allem aber von der Vereinigung für chinesisch-japanische Freundschaft (VCJF), außerordentlich liebenswürdig aufgenommen. Besonders die außerordentliche Ehre unseres Empfanges durch den Stellvertretenden Vorsitzenden des Ständigen Ausschusses des Volkskongresses, $\mathrm{Xu}$ Xiangqian, in der Großen Halle des Volkes zeigt die Beachtung, die man chinesischerseits dem japanischen Anwaltsverein schenkte.

Wie auch andere Besucher Chinas sahen wir Volksschulen, Luftschutzunterkünfte, Arbeiterwohnungen, einen Jugendpalast (a), Volkskommunen, eine Operation mit Akupunkturbetäubung usw. - Dinge, die uns tiefe und vielfältige Eindrücke hinterließen. Als Jurist möchte ich mich hier aber darauf beschränken, über eine Verhandlung in einer Strafsache zu berichten, wie wir sie als erste Japaner beobachten durften.

Leider bin ich nun freilich nur Anwalt, nur Praktiker. Ich bin ohne Vorkenntnisse über das chinesische Recht nach China gefahren. Als Laie im chinesischen Recht kann ich also hier nur wiedergeben, was ich mir, nach der Übersetzung unseres Dolmetschers, notiert habe. Dieser Bericht dürfte daher nicht wenige Irrtümer enthalten. Der sehr seltenen Gelegenheit, ein solches Verfahren zu beobachten, bewußt, habe ich mich aber bemüht, so gewissenhaft und objektiv, wie es mir nur möglich war, zu berichten, so daß das Folgende vielleicht doch der Aufmerksamkeit des Lesers wert sein mag.

\section{Erläuterungen vor der Verhandlung}

Wir hatten der VCJF, die uns betreute, unseren Wunsch ausgedrückt, unbedingt auch eine wirkliche Verhandlung und ein Gericht ${ }^{1}$ besuchen $\mathrm{zu}$ dürfen. Darauf erhielten wir Gelegenheit, am 11.12.1974 nachmittags einer Strafverhandlung

\footnotetext{
* Der Bericht ist erschienen in Hõritsu Jihõ Bd. 47 (1975), Nr. 568, S. 112. (Bei japanischen und chinesischen Namen steht der Familienname zuerst.) Die Úbersetzung besorgte Dr. Frank Münzel, Max-PlanckInstitut für ausl. u. intern. Privatrecht, Hamburg.

(a) Anmerkungen des Übersetzers - (a) bis (k) s. S. 370.

1 Gelegenheit, auch ein Gericht zu besichtigen, bot sich uns nicht. Als wir im Auto durch Shanghai fuhren, entdeckte ich an einer Ecke des zweiten Stocks eines Häuserblocks in einer Geschäftsstraße
} 
beizuwohnen. Dazu brachte man uns nicht in ein Gerichtsgebäude, sondern in ein Krankenhaus in Peking. (Um die Angeklagte zu schützen, lasse ich den Namen des Krankenhauses weg.) $\mathrm{Da}$ es um Diebstähle in diesem Krankenhaus ging, sollte der Volksgerichtshof der Mittelstufe der Stadt Peking heute dort an Ort und Stelle, vor den Leuten des Krankenhauses, das Verfahren erster Instanz durchführen².

Im Krankenhaus brachte man uns, bevor wir den Verhandlungsraum betraten, zunächst in ein Empfangszimmer. Dort gab uns der Vizepräsident des Volksgerichtshofs der Oberstufe der Stadt Peking, Herr Jiang Weixin, der uns schon auf dem Pekinger Flughafen empfangen und seither ständig betreut und uns bereits ausführliche Auskünfte über die Verhältnisse im chinesischen Gerichtswesen gegeben hatte, nun einige Erläuterungen zu dem heutigen Fall. - Herr Jiang war etwa 50 Jahre alt und wirkte milde und erfahren. Er war früher Anwalt.

Er erklärte uns: Die Angeklagte (wörtl. „zu Untersuchende“, shoushenren ${ }^{3}$ ) sei eine 45jährige Frau. Sie stamme aus einer Grundbesitzerfamilie im Kreise X in in der Provinz Y. Vor ihrer Festnahme habe sie in diesem Krankenhaus als Gehilfin verschiedene Arbeiten ausgeführt. Sie habe ihre der Ausbeuterklasse entsprechende Einstellung nicht geändert und sei zur Straftäterin geworden. Von 1966 bis September 1973 habe sie ihre Arbeit in der Wäscherei dazu benutzt, während der Abwesenheit anderer Angestellter zahlreiche Bettlaken, Bettbezüge, Patientenschlafanzüge u. a., insgesamt 1900 Teile im Wert von über 1600 RMB (etwa 256000 Yen [etwa $2400 \mathrm{DM}$ ]) $)^{4}$ zu stehlen (b). Sie sei auf frischer Tat ertappt und festgenommen worden. Sie habe ihre Beute weder verkauft noch sonst verwendet, abgesehen davon, daß sie etwas Gaze beim Dämpfen von Mantou [chines. Hefeklößen] verbraucht habe. Nach ihrer Festnahme habe sie die Diebstähle zugegeben, und ihre Haltung sei relativ gut gewesen; aber auch wegen der Gefühle der Massen solle hier zur Erziehung der Massen $^{5}$ nun das Verfahren öffentlich stattfinden, so daß es allen bekannt gemacht werde, alle Kritik äußern könnten. Bereits 1970 sei ein Diebstahl der Angeklagten entdeckt, aber, da sie (damals gleich) gestanden habe, ihr verziehen worden. Dennoch habe sie auch danach ihre Diebstähle fortgesetzt. Sie sei am 29.9.1973 verhaftet worden und seitdem im Haftverfahren (d. h. auf Grund eines Haftbefehls) im Polizeigefängnis in Haft gewesen, während die beteiligten Stellen eine Voruntersuchung ${ }^{6}$ durch-

ein Schild "Volksgerichtshof ", das sich aber von den üblichen Ladenschildern in keiner Weise unterschied. Wie uns gesagt wurde, hält man in China Gerichtsverhandlungen prinzipiell möglichst an Ort und Stelle ab. Ich nehme an, daß deshalb die Räumlichkeiten der Gerichte nur wenig benutzt werden und daher nur klein zu sein brauchen.

2 China hat ein vierstufiges Gerichtssystem: den Obersten Volksgerichtshof und Volksgerichtshöfe der oberen, mittleren und Grundstufe. Das Verfahren kennt zwei Instanzen. Die Stadt Peking hat einen Gerichtshof der Mittelstufe; er ist für die meisten Sachen die zweite und für wichtige Sachen die erste Instanz. Ob es dabei einfach darauf ankommt, daß eine Sache als wichtig angesehen wird, oder ob es noch andere Kriterien für die erstinstanzliche Zuständigkeit des Volksgerichtshofes der Mittelstufe gibt, haben wir zu fragen versäumt. (f)

3 Eine Person, gegen die eine Untersuchung läuft, wird als beigaoren (wörtl.: Angeklagter) bezeichnet; nach Anklageerhebung nennt man sie shoushenren (wörtl.: jemand, der überprüft wird). Der Einfachheit halber spreche ich hier durchweg von der Angeklagten.

4 Wie wir in einer Pekinger Volksschule hörten, lagen die Gehälter der Lehrer dort zwischen 40 und 80 RMB. 1600 RMB sind also eine beachtliche Summe.

5 Frau Zhang Fengge (Vizevorsitzende der Strafabteilung des Pekinger. Volksgerichtshofs der Mittelstufe), die uns das chinesische Strafverfahren erläuterte, sagte uns, bei den Strafverfahren unterscheide man Fälle von "Widersprüchen innerhalb des Volkes" und Fälle von Widersprüchen zwischen dem Feind und uns ${ }^{*}$. Gegenüber Feinden werde diktatorisch verfahren; bei Widersprüchen im Volke hingegen werde am Orte der Straftat gestützt auf die Massen Erziehung durchgeführt.

6 Nach Prof. Asai (Gendai Chūgoku hõ no riron [Die Theorie des heutigen chinesischen Rechts, Tokyo 1973], S. $127 \mathrm{f}$.) "müssen in China alle Fälle, in denen die Volksstaatsanwaltschaft Anklage verfolgung zu verhindern, die Korrektheit in den angeklagten Fällen $\mathrm{zu}$ er, eine unangemessene Strardaß sich die öffentliche Verhandlung auf ihren eigentlichen $Z$ weck, nämlich auf die rechtliche Lösung des Falles, konzentrieren kann. Die Voruntersuchung gehört nicht zur Verhandlung. Die Uuberprüfung 
geführt hätten. Am 31.10.1974 habe die Staatsanwaltschaft ${ }^{7}$ Anklage erhoben; daraufhin werde nun heute die öffentliche Verhandlung durchgeführt. Gemäß dem Gerichtsorganisationsgesetz könne die Angeklagte sich selber verteidigen oder sich eines Verteidigers bedienen. Im vorliegenden Fall habe die Angeklagte gestanden und auch nicht den Wunsch geäußert, einen Spezialisten ${ }^{8}$ als Verteidiger zu erhalten. Deshalb habe das Gericht ihr zwei Angestellte des Krankenhauses (einen Heilpraktiker [der chinesischen traditionellen Medizin] und eine Gehilfin) als Verteidiger zugewiesen, und die Angeklagte habe dem auch zugestimmt.

\section{Eröffnung des Verfahrens}

Nach diesen Erklärungen wurden wir zum Gerichtsraum geführt, nämlich zu einem sonst wohl für Vorlesungen dienenden Saal des Krankenhauses, in dem sich jetzt gut 200 Menschen drängten. Meist Angestellte des Krankenhauses waren darunter, besonders viele junge Mädchen, wohl Krankenschwestern. Später hörte ich, daß auch Studenten der juristischen Fakultät der Universität Peking unter den Zuhörern gewesen seien. Zusammen mit unseren Dolmetschern fanden

der Beweise auf ihre Glaubwürdigkeit usw., also die eigentliche Ưberprüfung und Regelung des Falles, darf in ihr nicht vorgenommen werden; auf Grund der Voruntersuchung soll lediglich entschieden werden, ob ein Fall, in dem ein Strafverfahren eingeleitet worden ist, in einer Gerichtsverhandlung überprüft und geregelt werden soll. ${ }^{\alpha}$ Mir scheint aber, daß in dem Fall, dessen Verhandlung ich sah, die Voruntersuchung anders durchgeführt wurde. Zwar habe ich leider, da ich damals von dem Problem der Voruntersuchung nichts wußte, die Beteiligten nicht danach gefragt, aber nach den Erklärungen, die man uns gab, klang es so, als ob in der Voruntersuchung bereits die Umstände und Motive der Tat, die Herkunft des Angeklagten, seine allgemeine Einstellung usw. untersucht würden; es schien der Tat, die Herkunft des Angeklagten, seine allgemeine Einstellung usw. untersucht würden; es schien
also, daß man hier durchaus schon in die Sache selbst eindrang. Auch nach Kita Kyöko (Peking Report; in Asia Review, Januar 1975) wird „die Voruntersuchung in China als sehr wichtig angesehen: in der Voruntersuchung werden die Beziehungen des Angeklagten zu seiner Umwelt, werden seine Einstellung und sein Denken eingehend untersucht; dabei wird selbstverständlich, schon bevor die Sache dem Gericht übergeben wird, auf die Wandelung der Einstellung und des Denkens (des Täters) auf Grund einer auf die Massen gestützten Erziehungsarbeit besonderes Gewicht gelegt. Unter Berücksichtigung der Meinung der Massen am Arbeitsplatz, der Meinung der Freunde, Eltern und Geschwister (des Angeklagten) ergeht dann schließlich das Urteil." - Vielleicht hat sich das Vorverfahren seit der Kulturrevolution geändert. (g)

$7 \mathrm{Kurz}$ nach unserer Reise, am 17. 1. 1975, verabschiedete der 4. Nationale Volkskongreß die neue chinesische Verfassung, nach deren Art. 25 die Funktionen und Befugnisse der staatsanwaltschaftlichen Organe von den Organen für öffentliche Sicherheit aller Ebenen ausgeführt werden, die also die Volksstaatsanwaltschaften abschaffte. Nach dem Bericht des Anwaltes Yamamoto Tadeyoshi, der kurz vor uns - im September 1974 - China besucht hat, sind seit der Kulturrevolution die Staatsanwaltschaften abgeschafft worden (Jiyu to seigi, Februar 1975). Wie das zu verstehen ist und was der Grund für die Abschaffung der Staatsanwaltschaften sein mag, darüber erhoffe ich die Meinung der Experten. Wenn ich als Laie mir eine Vermutung zum Grund der Abschaffung erlauben darf, so die, daß es die eigentliche Funktion der Staatsanwälte war, im Strafverfahren eine Strafe oder sonstige Regelung zu fordern; daß aber mit dem Erstarken der Massenlinie seit der Kulturrevolution mehr Gewicht auf die Voruntersuchung gelegt wurde, so daß also auch in bezug auf die Frage, wie mit dem Straftäter $\mathrm{zu}$ verfahren sei, die Voruntersuchung nun eine große Rolle spielt, während demgegenüber die Strafforderungsfunktion des Staatsanwaltes an Bedeutung verloren hat. Auch in der öffentlichen Verhandlung fordert der Staatsanwalt keine bestimmte Strafe, sondern läßt statt dessen die Massen das Strafmaß diskutieren, so daß also auch hier der Staatsanwalt seine Funktion verloren hat. Kurz, mir scheint, daß mit der völligen Durchführung der Massenlinie die Staatsanwaltschaft ihre Existenzberechtigung verloren hat und deshalb aufgelöst wurde. (h)

8 Nach der Erklärung, die uns Vizevorsitzender Jiang gab, sind nach dem Gerichtsverfassungsgesetz als Verteidiger nicht nur Anwälte zugelassen, sondern auch Verwandte und Freunde des Betroffenen, Bürger aus Staatsorganen, gesellschaftlichen Organisationen oder vom Arbeitsplatz (des Betroffenen), ebenso wie von gesellschaftlichen Organisationen empfohlene oder vom Gericht benannte Bürger. (i) 1956 sei ein vorbereitender Rechtsanwaltsausschuß gebildet worden. Damals habe es Anwälte im Hauptberuf und im Nebenberuf gegeben. Später aber sei man zu der Auffassung gelangt, daß die Notwendigkeit für die Existenz hauptberuflicher Anwälte weggefallen sei. Man habe sich im Gerichtsverfahren die Massenlinie zu eigen gemacht, man lege ein großes Gewicht auf die Untersuchung der Fälle und nur wenig Gewicht auf Geständnisse der Angeklagten; so habe man jetzt ein objektives Verfahren, in dem auch der Angeklagte selbst durchaus im Stande sei, sich zu verteidigen. Daher habe es nur noch wenig Aufträge und daher nur noch wenig Arbeit für Anwälte gegeben, so daß die Anwälte sich dann anderen Aufgaben gewidmet hätten; ein Teil sei zu den Gerichten gegangen. Er selbst sei ursprünglich Anwalt gewesen. Anwälte im Hauptberuf gebe es jetzt nicht mehr, wohl aber Anwälte im Nebenberuf. Ein Angeklagter könne zum Beispiel über das Gericht einen Professor oder Lektor der (juristischen Fakultät der) Universität Peking mit seiner Verteidigung beauftragen. 
wir in der Mitte rechts unsere Plätze. Für die Übersetzung bekamen wir Kopfhörer. $\mathrm{Da}$ wir gebeten worden waren, keine Bandaufnahmen oder Fotos zu machen, waren wir auf Notizen angewiesen.

Vorne vor der Versammlung befand sich eine Plattform. Links und rechts vor ihr standen je ein Polizeibeamter, mit dem Gesicht zu uns. Mit ihren umgeschnallten Pistolen wirkten sie recht furchteinflößend. Auf der Plattform saß in der Mitte der Vorsitzende Richter (shenpanyuan), ein vielleicht 45 Jahre alter Berufsrichter, der wie ein solider altgedienter Veteran wirkte ${ }^{9} . \mathrm{Zu}$ seinen Seiten saßen die Beisitzer (peishenyuan), ein Mann und eine Frau. Vor ihnen hingen am Tisch je ein Schild mit der Aufschrift „peishenyuan“. Links, mit dem Gesicht zum Vorsitzenden, saß ein Mann an einem Tisch mit dem Schild „shujiyuan“ (Protokollführer). Links von ihm saßen ein Mann und eine Frau, die ein Schild als Verteidiger auswies. Rechts saßen mit dem Gesicht zum Vorsitzenden zwei Männer, die nach ihrem Schild die öffentlichen Ankläger waren.

Auf einen Wink des Vorsitzenden hin riefen die Polizisten die Angeklagte herein, eine kleine dicke Frau, die sich vor den Richtertisch stellte. Der Vorsitzende erklärte die Verhandlung für eröffnet und fuhr fort: „Das Verfahren betrifft einen Fall von Diebstahl. Die Angeklagte heißt . .., hat den Beinamen ...., ist 45 Jahre alt, gehört zum Han-Volk (d. h. zu den Chinesen im engeren Sinne, nicht zu einer Minderheit), stammt aus einer Grundbesitzerfamilie, war selber Oberschülerin, hat das erste Jahr der Oberstufe der Mittelschule abgeschlossen; ihr Heimatort (d. h. der Ort, aus dem ihre Familie stammt, nicht unbedingt ihr Geburtsort) ist ... im Kreis ... in der Provinz ..." Sodann stellte er Fragen an die Angeklagte.

Aus deren Antworten ergab sich: Vor ihrer Festnahme hatte sie für $42 \mathrm{RMB}$ im Monat in der Wäscherei des Krankenhauses gearbeitet. Sie war nicht vorbestraft. Ihre Famile bestand aus ihrem 50jährigen Ehemann, einem Arbeiter mit 70 RMB Monatslohn, sowie drei Kindern: einem 17- und einem 15jährigen Mädchen, beide Oberschülerinnen und Mitglieder der Roten Garde, und einen 9jährigen Jungen, einem Volksschüler. Die Familie kam mit dem Einkommen der Ehegatten gut aus. Ihre Lebensumstände waren gut, und ihre Ersparnisse betrugen zeitweise 1400 RMB (etwa 224000 Yen [etwa 2000 DM]).

Darauf erklärte der Vorsitzende der Angeklagten: Nach Abschluß der vom Recht bestimmten Untersuchung werde die Angeklagte (nunmehr) hier der Anklage und der Massenkritik unterzogen. Sie habe das Recht, sich selbst zu verteidigen. Er gab dann die Teilnehmer am heutigen Urteilsverfahren bekannt: Das Verfahren werde vom Volksgerichtshof der Mittelstufe der Stadt Peking durchgeführt. Die eine Beisitzerin $^{10}$ (sie war etwa 20 Jahre alt) heiße . . . und sei Krankenschwester in diesem

9 Die Richter und Gerichtsvorsitzenden, denen wir in China begegnet sind, machten nicht, wie in der Regel der japanische Richter, den Eindruck von Intellektuellen, sondern sie wirkten meist mehr mit beiden Beinen im Leben stehend und welterfahren.

10 Den Inhalt dieses Artikels habe ich bereits in der "Studiengruppe Sozialismus“ am soziologischen Seminar der Universität Tokyo vorgetragen. Dabei haben die Professoren Ubukata Naokichi und Hariu Seikichi mich darauf hingewiesen, daß ursprünglich die Beisitzer gewählt bzw. ernannt wurden und dann für einen längeren Zeitraum ständig dem betreffenden Volksgerichtshof zugeordnet waren. Die Beisitzer in vorliegendem Fall indessen, die ja Leute waren, die in diesem Krankenhaus arbeiteten, dürften wohl nur für diesen Fall zu Beisitzern bestimmt worden sein. Auch die Bestimmung von Beisitzern mag sich also seit der Kulturrevolution geändert haben; mit anderen Worten, das Prinzip der Verhandlung an Ort und Stelle" ist auch im Verfahren der Bestimmung von Beisitzern durchgeführt worden. Hariu Seikichi hat übrigens in seinem Buch "Chūgoku no kokka to shakai“ (Chinas Staat und Recht), S. 117, ebenfalls eine Gerichtsverhandlung an Ort und Stelle beschrieben, wie sie 1958 in einer neuen Industriestadt stattfand; dieser Bericht hat mir das Verständnis der von mir beobachteten Strafverhandlung sehr erleichtert. 
Krankenhaus. Der andere Beisitzer (ein Mann mittleren Alters) heiße ... und sei Beamter im Sicherheitsdienst des Krankenhauses. Der eine Verteidiger (ein Mann mittleren Alters) sei Heilpraktiker in diesem Krankenhaus, die andere Verteidigerin (eine junge Frau) Gehilfin im Krankenhaus. Die Anklage sei von der Abteilung ... der Staatsanwaltschaft der Stadt Peking erhoben worden.

\section{Verlesung der Anklage, Befragung der Angeklagten}

Auf die Frage des Vorsitzenden, ob sie die Anklageschrift erhalten habe, antwortete die Angeklagte, sie habe sie vor drei Tagen erhalten.

Auf Aufforderung des Vorsitzenden verlas der Staatsanwalt die Anklage. Deren wesentliche Punkte waren: Die Angeklagte heiße mit Namen . . . und mit Beinamen ... ., gehöre zum Han-Volk und stamme aus einer Grundbesitzerfamilie; ihr Erziehungsgrad sei Oberstufe der Mittelschule; ursprüngliche Angestellte des Krankenhauses, sei sie am 29.9.1973 festgenommen worden und gegenwärtig in Haft. Bei der Angeklagten sei die Haltung der Ausbeuterklasse fest verwurzelt; sie habe eine große Menge von Behandlungsmaterial (des Krankenhauses) gestohlen; bereits einmal sei sie erzogen worden, indem die Massen sie kritisiert und ihr dann vergeben hätten; dennoch habe sie sich nicht gebessert, sondern ihre Diebstähle fortgesetzt. Sie habe Gaze, Saugwatte, Bettbezüge und anderes, insgesamt 1900 Stücke im Werte von über 1600 RMB gestohlen; deshalb werde sie heute vor dem Volksgerichtshof der Mittelstufe der Stadt Peking angeklagt.

Daraufhin erklärte der Vorsitzende, zur Angeklagten gewandt: Zur Erklärung des Rechts unseres Landes: Unser Land ist ein sozialistischer Staat unter der vom Vorsitzenden Mao geführten Diktatur des Proletariats. Gegenüber dem, der sich inmitten der Arbeiter (lebend und arbeitend) durch Arbeit an sich selbst bessert und ein Geständnis ablegt, wird Großzügigkeit geübt. Der Untersuchung und der Beweisaufnahme werden große Bedeutung beigemessen. Wenn der Straftäter die Sache, so wie sie (wirklich) war, gesteht, wenn er die seiner Straftat zugrundeliegende Einstellung überprüft, dann wird er großzügig behandelt. Ist er aber widerspenstig, dann wird er hart bestraft.

Nachdem der Vorsizende diese Erklärung gegeben hatte, erhob er, wie ich selber hören konnte, zur Angeklagten gewandt seine Stimme und sagte: „Gestehen Sie!"11

Die Angeklagte berichtete mit leiser Stimme: „Ich habe von 1966 bis 1973100 Bettücher, 50 Kilo Gaze und vieles andere, unter anderem Schlafanzüge, gestohlen. Meine Methode dabei war es, kleine Sachen in den Taschen zu verstecken."

Die junge Beisitzerin zeigte ihr ein Foto und fragte: „War das diese Tasche in dieser Hose?" "Ja", antwortete die Angeklagte.

11 Für uns, die wir von der Vorbereitung der Verhandlung durch das chinesische System der Voruntersuchung nichts wußten und das in Japan herrschende. "Prinzip einer einzigen Anklageschrift" (k) gewöhnt waren, war diese plötzliche Aufforderung des Vorsitzenden recht erstaunlich. 


\section{Beweisaufnahme}

Der Vorsitzende erklärte, man wolle nun die Aussagen der Zeugen hören. Darauf stand unter den Zuhörern sofort eine Frau auf und sagte aus: „Ich bin von diesem Krankenhaus. Eines Tages fiel mir auf, daß sie (die Angeklagte) sich unnatürlich verhielt. Ich ging dem nach und entdeckte versteckte Bettlaken. Als sie ertappt worden war, gestand sie den Diebstahl und bat um Verzeihung."

Auf Befragen des Vorsitzenden sagte die Angeklagte, sie habe die gestohlenen Sachen mit nach Hause genommen und dort versteckt. Etwas Gaze habe sie verbraucht, aber verkauft habe sie nichts. Ein Beisitzer verlangte Zeugen. Darauf stand ein Mann mittleren Alters auf und sagte aus: „Ich habe als Polizist (wörtlich: als Mann von der öffentlichen Sicherheit) den Ort (d. h. die Wohnung der Angeklagten) durchsucht. Aus Schränken (a) und Betten (a) wurde eine große Menge gestohlener Sachen hervorgeholt, so viel, daß ein Lastwagen damit beladen werden konnte. Der Beisitzer zeigte der Angeklagten Fotos (a) gestohlener Gegenstände. Die Angeklagte erkannte sie und berichtete dann, sie habe vorher auch wattierte Schlafröcke, chirurgische Messer und anderes gestohlen.

Auf die Frage des Vorsitzenden, warum sie den Weg des Verbrechens eingeschlagen habe, erwiderte die Angeklagte: „Ich entstamme der Ausbeuterklasse und bin unter dem Einfluß dieser Klasse aufgewachsen. Die Regierung hat uns Erziehung zur Wandlung unseres Denkens gewährt, aber ich habe die Wandlung meines Denkens und meine politischen Studien vernachlässigt; seit meiner Schulzeit habe ich meine auf persönlichen Erfolg im Leben ausgerichtete individualistische Einstellung nicht geändert. Ich habe mich den Vorstellungen der Proletarischen Kulturrevolution widersetzt; all das ist meine Schuld. Während der zweiten Hälfte der Proletarischen Kulturrevolution bin ich von den Massen kritisiert, aber großzügig behandelt worden. Doch das nutzte alles nichts. Ich habe den Massen und der Führung gegenüber keine Entschuldigung. In einem Satz gesagt: Daß ich so den Weg des Verbrechens beschritten habe, liegt am bürgerlichen Denken."

Auf Fragen der Anklage erklärte die Angeklagte, die Diebstähle hätten sich über einen Zeitraum von über 8 Jahren erstreckt; sie habe sich nicht selbst angezeigt, sondern sei von den Massen gefaßt worden; das gestohlene Gut sei entdeckt und beschlagnahmt worden.

Auf Fragen der Verteidigung sagte die Angeklagte, sie habe schon vor der Festnahme teilweise gestanden, das meiste aber erst nach der Festnahme; sie sei nicht vorbestraft.

\section{Plädoyer}

Auf Aufforderung des Vorsitzenden trug die Anklage ihr Plädoyer vor, wie folgt: „Der Tatbestand der Straftrat ist durch sachliche Beweise und durch Zeugen in Gänze bewiesen. Die Angeklagte gibt ihn auch zu. Nach der Verfassung unseres Landes ist das Volkseigentum heilig; die Pflicht der Angeklagten war es vor allem, das Staatsvermögen zu schützen. Dennoch hat sie seit 1966 ihre dienstliche Stellung zu Diebstählen benutzt. 1970 ist sie bereits einmal ertappt und kritisiert worden, aber es wurde ihr verziehen. Dennoch hat sie ihre Diebstähle fortgesetzt und bis zum 29. 9. 19731900 Gegenstände im Wert von über 1600 RMB gestohlen. 
Die Leute in diesem Krankenhaus sind erzürnt; ich beantrage somit ein korrektes Urteil.“ (Die Anklage stellte keinen Strafantrag.)

Darauf stand einer der Verteidiger auf und plädierte wie folgt: „Ich habe mich mit der Angeklagten zusammengesetzt und ausführlich mit ihr gesprochen. Die Tatsachen der Anklage sind nicht zu bestreiten. Aber aus folgenden Gründen beantrage ich eine milde Behandlung (der Angeklagten).

(1) Die Angeklagte hat den Sachverhalt zugegeben. Sie ist sich selbst auch bewußt, daß sie angesichts ihrer Herkunft aus der Ausbeuterklasse ihr bürgerliches Denken nicht hat korrigieren können.

(2) Sie hat nach ihrer Festnahme ihre Straftat zugegeben und ein relativ gutes Verhalten gezeigt.

(3) Sie hat nur einen Teil der gestohlenen Sachen in Gebrauch genommen und nichts verkauft.

(4) Die gestohlene Menge ist nicht groß (sic!), die Angeklagte ist nicht vorbestraft. Von gewohnheitsmäßigem Diebstahl (c) kann man daher nicht sprechen.

Wenn, wie geschildert, der Straftäter ein Geständnis ablegt, so ist er, wie uns Vorsitzender Mao lehrt, gemäß der Politik der KP Chinas großmütig zu behandeln; daher bitte ich um ein großmütiges Urteil gegenüber der Angeklagten."

Darauf stand nochmals ein Ankläger (a) auf und sagte, die Angeklagte habe tatsächlich schon vor ihrer Verhaftung einiges zugegeben; aber das sei keine Selbstanzeige gewesen, sondern sie habe gestanden, weil man sie ertappt habe. Auch ihre Beute habe sie nicht von sich aus herausgegeben. Es sei das etwas anderes, als wenn sie von sich aus gestanden und der allgemeinen Kritik sich unterworfen hätte.

Darauf erklärte die Verteidigung, die Angeklagte habe sich zwar nicht selbst angezeigt, aber als man sie ertappt habe, da habe sie ihre Straftat so, wie sie wirklich gewesen sei, zugestanden. Das sei als Selbstanzeige anzusehen (d). Sie habe zwar einen Teil der Beute benutzt, aber den größten Teil weder zerstört (= verbraucht) noch verkauft.

Der Ankläger (a) stand wiederum auf und erklärte, zum Vorsitzenden gewandt, er habe den Einwänden der Verteidigung nichts hinzuzufügen; man möge die Meinung der Massen hören und achten.

\section{Die Meinung der Massen}

Der Vorsitzende ließ nun die Angeklagte sich so hinstellen, daß sie sich den "Massen“ zuwandte (da die Zuhörer auch am Verfahren teilnahmen, scheint es angebracht, von „den Massen“ statt von Zuhörern zu sprechen) und sagte dann zu den Massen: „Die Vertreter der Massen mögen die Hand heben!“

Sogleich hob eine Anzahl von Männern und Frauen einer nach dem anderen die Hand. Auf Aufruf des Vorsitzenden hin standen sie auf und sagten ihre Meinung. Sie sprachen alle recht klar und ausführlich; aber meine Fähigkeiten als Protokollführer reichten nicht aus, alles mitzuschreiben; ich gebe hier nur die wesentlichen Punkte wieder.

Ein etwa 50jähriger Mann: Die Angeklagte war ursprünglich in der Buchhaltung beschäftigt. Aber weil sie ihre Stellung zu ihrer Bereicherung benútzte, ist sie in die Wäscherei versetzt worden. Aber überall stiehlt sie. Zu ihrer Diebesbeute 
gehören Toilettenpapier, Operationsgeräte usw. - man kann es gar nicht alles aufzählen. Auch Schaufeln und Handschuhe hat sie gestohlen. Und obwohl man ihr beim ersten Mal verziehen hat, hat sie weiter gestohlen. Die Angeklagte wendet sich gegen den Sozialismus.

Eine etwa 20jährige Frau: Aus ihrer bösen Natur heraus hat die Angeklagte auch schon 1970 während der Proletarischen Kulturrevolution gestohlen. Damals haben wir eine Versammlung abgehalten und die Sache diskutiert. Da die Angeklagte bereute, sind wir großmütig mit ihr verfahren. Aber sie hat ihre Diebstähle fortgesetzt. Die Angeklagte zerstört die Diktatur des Proletariats. Ihre Reue damals war eine Lüge.

Noch eine etwa 20jährige Frau: Die Angeklagte stammt aus der Ausbeuterklasse. Sie ist reaktionär erzogen. Sie hat einen ganzen Lastwagen voll gestohlen. Sie hat eine schlechte Natur.

Eine etwa 30jährige Frau: Die wahre Gestalt der Angeklagten ist jetzt ganz deutlich geworden. Sie ist ein reaktionäres Element. Sie hat fortgesetzt gestohlen. Obgleich sie doch damals großzügig behandelt worden ist, hat sie noch schlimmer gestohlen als damals, und zwar, weil sie von Grund auf ein reaktionäres Element ist.

Ein etwa 45jähriger Mann (nachdem er mit lauter Stimme den Sinn der Diktatur des Proletariats erklärt hatte): Um die finsteren Machenschaften des Revisionismus zu zerschlagen, ist eine strenge Strafe für die Angeklagte zu fordern.

Eine etwa 18jährige Frau sprach über die Kritik an Lin Biao und Konfuzius.

Eine etwa 25jährige Frau: Die Angeklagte ist mit der Diktatur des Proletariats unzufrieden. Sie ist genauso wie Lin Biao und Konfuzius.

Eine etwa 35jährige Frau: Daß die Angeklagte zum Volksfeind geworden ist, das ist kein Zufall. Jeder steht unter dem Einfluß seiner Klasse. Die Angeklagte ist durch ein luxuriöses Leben zum Volksfeind geworden.

Nach Abschluß dieser Erklärungen der Massen ließ der Vorsitzende die Angeklagte, die mit gesenktem Kopf die Kritik der Massen angehört hatte, sich wieder ihm zuwenden und fragte sie: „Hat die Angeklagte etwas zu sagen?“ Darauf antwortete die Angeklagte: „Gegenüber Ihnen allen, von der Regierung (d. h. den Behörden) und vom Krankenhaus, habe ich keine Entschuldigung. Ich habe mich durchweg falsch verhalten. Mein bürgerlich-grundbesitzliches Denken ist nicht reformiert. Von nun ab werde ich gründlich an meiner eigenen Wandelung arbeiten, ich werde mich im proletarischen Denken festigen, ich möchte ein Mensch werden, der dem Proletariat nützt. Ich bitte, mir dazu Gelegenheit zu geben. Ich möchte die mir von Partei und Regierung erwiesene Gnade vergelten.

\section{Pause, Massendiskussion}

Der Vorsitzende erklärte nun eine Pause. Er ließ die Angeklagte sich zurückziehen und kam dann ohne weiteres von der Plattform herab. Seine eben noch strenge Haltung war plötzlich ganz verwandelt. Freundlich lächelnd sagte er: „Während der Pause können wir uns ein wenig über verschiedene Dinge unterhalten. Vorsitzender Mao lehrt uns, daß Fragen mit der Massenlinie zu lösen sind. Es gibt auch Punkte, die für eine günstige Beurteilung der Haltung der Angeklagten sprechen. Wie ist Ihre Meinung dazu?" 
Die Atmosphäre in der Versammlung milderte sich darauf sichtlich. Mehrere Leute standen einer nach dem anderen auf und sagten ihre Meinung. Die Hauptpunkte waren wie folgt:

Eine junge Frau: Die Angeklagte sollte inmitten der Massen gebessert werden (d. h. nicht in Strafhaft). Ein Mann mittleren Alters forderte drei Jahre Zwangsarbeit. Ein etwa 35jähriger Mann: Eine strenge Strafe ist schon notwendig. Aber sie hat nach der Festnahme gestanden. Das Diebesgut hat sie nicht verkauft, es konnte zurückbeschafft werden. Deshalb erscheinen mir zwei Jahre Zwangsarbeit angebracht. Eine etwa 20jährige Frau: Wenn sie zwei Jahre wirklich verbüßen soll, ist das zu streng. Man kann sie zu zwei Jahren verurteilen, aber die Vollstreckung aussetzen und sie unter Kontrolle der Massen (eine Art Bewährungsaufsicht) stellen. Eine andere etwa 20jährige Frau sprach sich auch für 2 Jahre Zwangsarbeit aus. Ein über 50jähriger Mann: Da die Haltung der Angeklagten gut ist, sollte man sie unter Massenkontrolle stellen. Eine etwa 20jährige Frau: 2 Jahre Zwangsarbeit (zur Bewährung ausgesetzt) und Überwachung durch die Massen wäre richtig. Eine Frau mittleren Alters: Zwei Jahre Zwangsarbeit, aber ohne daß sie in Haft genommen wird.

Nachdem es sich diese Erklärungen angehört hatte, zog sich das Gericht auf Veranlassung des Vorsitzenden zurück. Ihm folgend begaben auch wir uns in das Empfangszimmer des Krankenhauses.

\section{Beratung}

Im Empfangszimmer waren nun außer dem Vorsitzenden und den zwei Beisitzern noch der Leiter des Revolutionskomitees (RK) ${ }^{12}$ des Volksgerichtshofes der Mittelstufe der Stadt Peking, der Leiter des RK des Krankenhauses, der uns betreuende Vizevorsitzende des Volksgerichtshofes der Oberstufe der Stadt Peking, Herr Jiang Weixin, die Vizevorsitzende der Strafabteilung des Volksgerichtshofes der Mittelstufe, Frau Zhang Fengge, und wir versammelt.

Zunächst erstattete der Vorsitzende (des entscheidenden Gerichts) dem Leiter ${ }^{13}$ des RK des Volksgerichtshofes (der Mittelstufe) Bericht über den Verlauf der Verhandlung ${ }^{14}$. Als Meinung der Massen gab er wieder, man habe teils zwei, teils drei Jahre Strafarbeit gefordert; es sei sowohl die Ansicht vertreten worden, die Angeklagte solle in Haft genommen werden, als auch die, sie solle von der Haft verschont werden. Seine eigene Meinung sei, die Angeklagte solle zu zwei Jahren Strafarbeit verurteilt, aber nicht in Haft genommen, sondern im Krankenhaus unter den Massen belassen werden. Der Leiter des RK hielt auch dafür, daß Massen-

12 In China werden alle Einheiten - seien es Gerichte, Volksschulen, Krankenhäuser, Universitäten, Gebietskörperschaften usw. usw. - von Revolutionskomitees geleitet.

$13 \mathrm{Er}$ wirkte nicht wie ein Rechtsspezialist, sondern eher wie jemand, der aus der Arbeiterschaft kam. Einen ähnlichen Eindruck hatte ich auch von den Leitern der Revolutionskomitees des Universitätskrankenhauses und sonst an der Pekinger Universität, die wir kennenlernten. Auch sie schienen nicht zu den Spezialisten dieser Einrichtungen zu gehören, sondern eher Leute der revolutionären Bewegung bzw. Arbeiter zu sein.

14 Frau Zhang Fengge erklärte uns: Seit der Kulturrevolution sei man auf Grund der Entwicklung der Massenlinie dazu gekommen, daß außer den drei das beratende Gremium bildenden Richtern nun auch von Fall zu Fall andere mit der Justiz in Verbindung stehende Personen an der Beratung teilnähmen. Wenn die Beratung zu einem Ergebnis gelangt sei, müsse dieses nun auch der Leitungsgruppe (dem RK) gemeldet werden, ebenso wie dem Gericht der nächsthöheren Stufe. Deshalb trug im vorliegenden Fall auch Frau Zhang ihre Meinung zu dem Fall vor, und den Leitern des RKs des Gerichts und des RKs des Krankenhauses wurde Bericht erstattet. Man mag die Sache sogar so ansehen: den Leitern der RKs wurde nicht nur Bericht erstattet, sondern mit der Erklärung, sie hätten nichts einzuwenden, gaben sie auch ihre Meinung kund und erkannten den Bericht an. 
kontrolle angebracht sei, fragte aber, von wann an die Strafe gerechnet werden solle. Der Vorsitzende meinte, vom Tage der Inhaftierung der Angeklagten, also dem 29. 9. 1973 an; der Leiter war einverstanden. Der Vorsitzende gab als weitere Gründe für die Aussetzung der Strafe an, daß die Angeklagte ein kleines (9jähriges) Kind habe; daß man ferner auch das Verhältnis der Angeklagten zu ihren beiden älteren (17 und 15 Jahre alten) und der Mutter gegenüber kritisch eingestellten Kindern bedenken müsse; und daß schließlich die Haltung der Angeklagten gut sei. Dann sagte die Vizevorsitzende Zhang Fengge, die Angeklagte habe zwar aus Habgier, entsprechend dem Denken der Ausbeuterklasse, ihre Straftat begangen, aber ihre Haltung nach der Inhaftierung sei gut gewesen; eine Strafe von zwei Jahren, ohne Strafhaft und mit Unterstellung unter Bewährungsaufsicht am Arbeitsplatz sei das Richtige. Dabei sei auch zu berücksichtigen, daß die (gesetzlichen) Bestimmungen bei einer Beute von unter 5000 RMB 1 bis 5 Jahre Zwangsarbeit vorsähen (e).

Dann bat der Vorsitzende die zwei Beisitzer und den Leiter des RK des Krankenhauses um ihre Meinung; sie stimmten alle mit der Meinung des Vorsitzenden überein.

\section{Fragen der Besucher}

Nachdem somit die Beratung abgeschlossen war, wurden Fragen erlaubt. Wir stellten folgende Fragen:

Von der Festnahme bis zur heutigen Verhandlung sind $1 \mathrm{Jahr}$ und drei Monate vergangen. Warum hat man so lange gebraucht? Ferner ist die Strafe von zwei Jahren zwar ausgesetzt worden; dadurch aber, daß die Angeklagte bereits $1 \mathrm{Jahr}$ und 3 Monate in Haft gewesen ist, ist die Strafe doch tatsächlich schon zum guten Teil vollzogen worden; wie denkt man darüber?

Vizevorsitzender Jiang vom Volksgerichtshof der Oberstufe antwortete: Daß man bis zur Verhandlung ein Jahr und drei Monate gebraucht habe, hänge damit zusammen, daß sich die Straftaten der Angeklagten seit 1966 über einen Zeitraum von 8 Jahren erstreckt hätten, so daß die Untersuchung und Vorprüfung seitens der zuständigen Behörden auch längere Zeit beansprucht habe. Was den Zweifel anbelange, ob man die Strafe nicht praktisch schon als teilweise verbüßt ansehen müsse, so sei eben dieser Punkt auch mit in Erwägung gezogen worden, als man beschlossen habe, die Strafe auszusetzen.

Wir fragten, ob eine Entlassung gegen Sicherheit möglich sei. Darauf hieß es, wer gesund sei, könne vor dem Urteil nicht entlassen werden.

Ferner wurde uns erläutert, nach dem Urteil werde je ein Exemplar des Urteils dem Krankenhaus und dem Polizeiposten (paichuso) zugeschickt, zur Überwachung der Angeklagten. Mache die Angeklagte gute Fortschritte, so werde das RK des Krankenhauses, sich auf die Meinung der Massen stützend, mit dem Polizeiposten beraten und (dann) dem Gericht eine Strafsenkung vorschlagen. Im gegenteiligen Falle werde das Verfahren der Aufnahme in die Strafhaft durchgeführt. 


\section{Urteil}

Nachdem somit unsere Fragen erledigt worden waren, kehrten wir zusammen in den Gerichtsraum zurück.

Der Vorsitzende erklärte die Verhandlung für wieder eröffnet und rief die Angeklagte herein. Er ließ sie sich vor ihm aufstellen und verkündete dann folgendes Urteil:

„Dies Gericht hat die Anklage der Abteilung der Staatsanwaltschaft der Stadt Peking in der Sache gegen ... (Name der Angeklagten) erhalten. Darauf ist eine eingehende Untersuchung durchgeführt worden. Bei der heutigen öffentlichen Verhandlung ist die Sache nochmals überprüft und erwogen worden. Sowohl die Umstände der Straftat als auch die Haltung der Angeklagten sind erwogen, auch die Meinung der Angeklagten ist gehört worden. In der kollektiven Beratung haben wir auch die Leitung des Krankenhauses (das bedeutete, wie uns erklärt wurde, die Meinung des RK des Krankenhauses) und unsere Leitung (das heißt, die Meinung des RK des Gerichtes) gehört und wie folgt entschieden:

Die Untersuchung der Zelle für Strafsachen des Volksgerichtshofes der Mittelstufe der Stadt Peking betrifft die Angeklagte ..., weiblich, 45 Jahre alt, Angehörige des Han-Volkes, aus einer Grundbesitzerfamilie stammend, selber Oberschülerin; ihr Heimatort ist ... im Kreis . . . in der Provinz . . .; ihr gegenwärtiger Wohnsitz ist . . . Wegen Diebstahl ist sie am 29.9.1973 festgenommen worden. Als Ergebnis der Untersuchung wird festgestellt, daß sie folgende Straftaten begangen hat:

Die Angeklagte hat bereits 1966 Diebstahl begangen, aber von 1966 an sind die Diebstähle besonders schlimm geworden. Sie hat ihre dienstliche Stellung benutzt, um Diebstähle in großem Ausmaß zu begehen. 1970 wurde sie ertappt. Sie wurde von den Massen kritisiert, aber man verzieh ihr. Dennoch hat sie ohne zu bereuen auch danach noch ihre Diebstähle fortgesetzt. Sie hat $45 \mathrm{~kg}$ Gaze, $25 \mathrm{~kg}$ Verbandwatte $^{15}$, Bettücher, Bettbezüge und andere medizinische Bedarfsgegenstände gestohlen, insgesamt 1900 Teile im Werte von über 1600 RMB. Fast das gesamte Diebesgut konnte beschlagnahmt werden. Die Angeklagte hat die Umstände der Straftat in Gänze zugegeben. Die Angeklagte stammt aus einer Grundbesitzerfamilie; sie hat Diebstahl in großem Ausmaß begangen, und das muß, um die sozialistische Ordnung zu schützen, streng bestraft werden. Die Haltung der Angeklagten ist jedoch gut. Somit wird folgende Strafe verhängt.

Die Angeklagte wird zu 2 Jahren $Z$ wangsarbeit verurteilt. Aber die Angeklagte wird nicht in Haft genommen, sondern hier im Krankenhaus der Aufsicht der Massen unterstellt. Die Strafe wird vom 29. 9. 1973 an gerechnet.

Nachdem er dieses Urteil verkündet hatte, erklärte der Vorsitzende die Verhandlung für geschlossen und wies die Polizisten an, die Angeklagte abzuführen. (Ich beobachtete das Gesicht der Angeklagten, als sie den Gerichtsraum verließ, konnte aber keinen besonderen Ausdruck darauf erkennen.) Auch wir verließen den Gerichtsraum.

15 Diese konkreten Zahlen fanden sich weder in der Anklage, noch ergaben sie sich aus den Zeugenaussagen und sonstigen Beweisen. Man fragt sich, warum sie jetzt im Urteil auftauchten. Vielleicht hatten sich diese Zahlen bereits in der Voruntersuchung herausgestellt, und man hielt es deshalb für überflüssig, sie jetzt in der öffentlichen Verhandlung nochmals eigens einzubringen. 


\section{Gedanken und Gefühle}

So endete also unser Besuch bei einer Gerichtsverhandlung. Im folgenden möchte ich noch meine Gedanken und Gefühle dazu wiedergeben.

(1) Von dem chinesischen "Volksgerichtsverfahren" hatte ich zwar schon manches gehört, aber hier hatte ich zum ersten Mal Gelegenheit, es in praxi zu sehen; und es ist wohl nur natürlich, daß mich seine - verglichen mit unserem japanischen Strafverfahren - völlige Andersartigkeit nicht wenig verwunderte. Aber trotz dieser Unterschiede war es doch so, daß wenn man das Ergebnis, nämlich das Urteil, betrachtete, dieses wohl durchaus angemessen war und bei dem gleichen Fall in Japan auch nicht viel anders ausgefallen wäre. Daß das Rechtsgefühl der chinesischen „Massen“ einerseits und das Rechtsgefühl von uns japanischen Juristen andererseits sich unerwartet wenig unterschied, diese Entdeckung erleichterte uns doch sehr. Zur Erzielung dieses Ergebnisses durch die „Massen“ hatte ich freilich den Eindruck, daß sich die Massen hier nicht völlig frei entfalteten, sondern sie vom RK und von dem erfahrenen Richter hinreichend geführt wurden. Diese starke Führung durch das (jeweilige) RK und die hinter ihm stehende KP haben wir bei allen unseren Besuchen in Schulen, in der Pekinger Universität, in Krankenhäusern und Volkskommunen sehr deutlich gespürt und daran immer wieder gesehen, daß sich China noch immer in der Revolution befindet, oder vielmehr, in den Worten Maos, in der fortgesetzten Revolution. Auch bei diesem Gerichtsverfahren hatten wir den Eindruck, daß man es als ein Verfahren im Strome der Revolution verstehen und erklären müsse.

(2) Ich hätte gerne gewußt, was denn die Angeklagte nun davon hielt, daß man sie an ihren ursprünglichen Arbeitsplatz zurückschickte - ob sie sich darüber freute, oder ob nicht eher das Gegenteil der Fall war. In Japan halten wir Anwälte es allgemein für richtig, bei Strafaussetzung den Angeklagten nicht an seinem ursprünglichen Arbeitsplatz, sondern an einem neuen Arbeitsplatz arbeiten $\mathrm{zu}$ lassen und seine Vorstrafe vor den Augen seiner Umwelt zu verbergen, damit $\mathrm{ihm}$ Gelegenheit gegeben wird, ein neues Leben zu beginnen. In China dagegen schickt man die Angeklagte an ihren alten Arbeitsplatz zurück, wo sie von den Massen mit Verachtung oder doch jedenfalls mit besonderen Augen angesehen wird; ich frage mich, ob ihr das nicht zuwider ist, ob sie nicht etwa statt dessen lieber die restlichen elf Monate ihrer Strafe abgesessen hätte. Deswegen habe ich auch versucht, nach dem Ende der Verhandlung den Gesichtsausdruck der Angeklagten $\mathrm{zu}$ erkennen. Als ich diese Frage gegenüber Teilnehmern an der Verhandlung ansprach, wurde mir gesagt, in China sehe man jemand, der seine Fehler eingesehen habe, keineswegs mit Verachtung an; vielmehr werde man die Angeklagte ganz entschieden und auf das wärmste als Genossin beim sozialistischen Aufbau betrachten und ihr dabei helfen, ein neues Leben zu beginnen, so daß es gar nichts gebe, was der Angeklagten zuwider sein könne. Mir scheint jedoch, daß dieser Punkt, auch wenn man die Unterschiede der Gesellschaftsordnung in Betracht zieht, dennoch zweifelhaft bleibt; es mag das auch mit den Unterschieden des traditionellen Schamgefühls der Chinesen einerseits, der Japaner andererseits zusammenhängen - wie dem auch sei: mir als Japaner scheint jedenfalls, daß man sich davor hüten sollte, hier die Gefühle der Angeklagten allzu leicht zu nehmen. 
(3) Die Angeklagte hat hier fortgesetzt gestohlen, und zwar eine große Anzahl von Dingen; sie hat aber nichts davon verkauft. Bei diesem Sachverhalt liegt ein krankhaftes Motiv für die Diebstähle nahe. Diese Frage ist jedoch überhaupt nicht aufgegriffen worden. Vielmehr wurde nur das „reaktionäre Denken“ der Angeklagten als Motiv angesehen und angegriffen. Das schien mir problematisch. Hätte es sich um ein japanisches Verfahren gehandelt, so hätte der Anwalt sich bei einem solchen Kleptomanen ausführlich zu der „kranken Psyche“ seines Mandanten geäußert - wenn dieser Punkt nicht bereits im Vorverfahren geklärt worden wäre.

\section{Schluß}

Wir konnten uns nicht nur durch den Besuch dieses Strafverfahrens über das chinesische Rechtswesen informieren; Herr Jiang Weixin, Frau Zhang Fengge und Frau Wang Shuwei (Vizevorsitzende der Zivilkammer des Volksgerichtshofes der Mittelstufe der Stadt Peking) haben uns ausführliche Erklärungen über das System der chinesischen Justiz und über die Verhältnisse in Straf- und Zivilverfahren gegeben. Von den Lehrkräften der juristischen Fakultät der Universität Peking haben wir einiges über die Praxis der juristischen Ausbildung erfahren. Kurz vor unserem Abflug konnten wir auch noch ein Gefängnis der Stadt Shanghai besichtigen. - Schon eine Stunde nachdem unser Flugzeug Shanghai verlassen hatte, tauchten unter uns die Klippen der japanischen Küste bei Miyazaki und Aoshima in den Wellen auf; von da ab bis Tokyo waren es dann nur noch anderthalb Stunden. So nahe bei Japan führen die 800 Millionen Chinesen ein so völlig andersartiges Leben - ein Leben, über das uns Japanern fast nichts bekannt ist. Mir erscheint das von großer Bedeutung. Im Flugzeug ging mir das immer wieder durch den Kopf. Ich halte es deshalb für meine Pflicht, über das, was ich bei diesem Verfahren und sonst im juristischen Bereich gesehen und gehört habe, den Juristen und der Öffentlichkeit Japans möglichst konkret zu berichten.

Allerdings ging es bei dem von uns beobachteten Verfahren um „Widersprüche im Volke“, nicht um ein „konterrevolutionäres“ Delikt. Außerdem waren in diesem Fall die Tatsachen unstreitig. Mir scheint, daß das Gerichtsverfahren seinen wahren Charakter in Fällen zeigt, in denen die Tatsachen streitig sind, und in konterrevolutionären Fällen, in denen das Interesse der Klassen gegeneinander steht. Daher scheint es mir gefährlich, allein von dem kleinen Diebstahlsfall aus, in den wir einen Einblick tun konnten, das chinesische Gerichtsverfahren zu beurteilen. - Davon ausgehend habe ich mich bemüht, meine Eindrücke und Urteile möglichst zurückzudrängen und einen Bericht zu geben, der nicht mit meinen subjektiven Ansichten vermischt ist.

Wenn ich dennoch am Schlusse dieses kleinen Berichts etwas nennen möchte, das mir einen bleibenden Eindruck hinterlassen hat, so ist es der warme und freundliche "Massencharakter", den wir bei den Leuten von den chinesischen Gerichten gespürt haben. Das gilt ebenso für Herrn Jiang und die beiden anderen vorerwähnten Richter, die wir in Peking kennenlernten, wie für Frau Lu Feng'e, mit der wir in Suzhou sprachen, der Vizevorsitzenden des Volksgerichtshofes der Mittelstufe dieser Stadt, die mehr wie eine freundliche Nachbarin als eine Richterin wirkte, und für Herrn Lou Jiating, den wir in Shanghai kennenlernten, 
den Vorsitzenden des Volksgerichtshofes der Oberstufe der Stadt Shanghai, der wie ein freundlicher Großvater aus der Nachbarschaft schien. Sie alle gaben einem keineswegs den Eindruck machtbewußter Beamter; sie strahlten vielmehr eine warme „Massenhaftigkeit“ aus.

Art. 25 der neuen chinesischen Verfassung bestimmt: „In der staatsanwaltschaftlichen Tätigkeit wie bei der Verhandlung von Rechtsfällen muß die Massenlinie durchgeführt werden." Man kann sagen, daß in dem Auftreten dieser Menschen, die wir (bei den chinesischen Gerichten) kennengelernt haben, die Massenlinie ihren konkreten Ausdruck gefunden hat, daß sich hier der Grundcharakter auch des chinesischen Verfahrens zeigt.

\section{Schlußbemerkung}

Den Professoren Ubukata und Hariu bin ich für zahlreiche Hinweise anläßlich des oben erwähnten Vortrags des wesentlichen Inhalts dieses Aufsatzes zu Dank verpflichtet.

Anmerkungen des Übersetzers

(a) Im Japanischen werden Singular und Plural meist nicht unterschieden. In der Ubersetzung habe ich jeweils eingesetzt, was mir am wahrscheinlichsten erschien.

(b) Vgl. hierzu und zum Folgenden die einschlägigen. Vorschriften des materiellen chinesischen Strafrechts in den Zhonghua renmin gongheguo chengzhi tanwu tiaoli (Regeln der VR China über die Bestrafung von Amtsuntreue, v. 21. 4. 1951, Zhongua renmin gongheguo fagui xuanji, S. 263; Amtsuntreue ist das widerrechtliche Ansichbringen von Staats- und genossenschaftlichem Eigentum; auch der Diebstahl der Angeklagten fällt also unter diese Vorschriften):

$\int 3$ III: Liegt der Wert der von dem einzelnen (Täter) veruntreuten Dinge zwischen 1000 neuen RMB und 5000 neuen RMB, so wird (die Tat). mit 1 bis 5 Jahren Freiheitsstrafe oder 1 bis 4 Jahren Arbeitslager oder 1 bis 2 Jahren Kontrolle (einer Art Bewährungsaufsicht) bestraft.

$\oint$ : Liegt bei Amtsuntreue einer der folgenden Umstände vor, so ist ein schwerer Fall gegeben bzw. kann die Strafe verschärft werden:

6. Trotz mehrerer Vorstrafen (lüfan) bessert sich der Täter nicht.

7. Der Täter weigert sich zu gestehen, oder er hindert andere am Geständnis.

10. Das Geständnis geht nicht bis ins Letzte, und nach der Verurteilung werden noch schlimmere Umstände entdeckt.

\$ 5:... (In den folgenden Fällen kann die Strafe gemildert oder der Fall statt durch Kriminalstrafe administrativ geregelt werden:)

1. Vor der Entdeckung der Tat gesteht der Täter aus eigenem Antrieb.

2. Nach der Entdeckung der. Tat gesteht der Täter alles bis ins Letzte, bereut wirklich und gibt aus eigenem Antrieb, soweit es ihm irgend möglich ist, die veruntreuten Gegenstände wieder heraus.

Diese Vorschriften erklären, warum im Folgenden Art und Umfang des Geständnisses der Angeklagten und die Modalitäten der Beschlagnahme der gestohlenen Gegenstände so betont werden.

(ba) Vgl. auch unten Anm. g.

(c) "Gewohnheitsmäßiger Diebstahl“" ist ein Terminus des japanischen Rechts, vgl. etwa $\ 2$ des japanischen Gesetzes über die Bestrafung von Eigentumsdelikten; er hat dort die gleiche Bedeutung wie bei uns. Der Dolmetscher des Verfassers dürfte mit diesem Ausdruck aber das chinesische "üfan" übersetzt, also "mehrere Vorstrafen" gemeint haben (vgl. oben Anm. b in $\$ 3$ III Nr. 6 der dort zitierten "Regeln“.) Nur so wird diese Bemerkung der Verteidigung verständlich.

(d) Auch hier liegt wohl eine ungenaue Ubersetzung vor. Gemeint ist wohl: „Das wird einer Selbstanzeige gleichgestellt" (nämlich in $\ 5 \mathrm{Nr} .2$ der in Anm. b zitierten "Regeln").

(e) Vgl. $\$ 3$ III der in Anm. b zitierten "Regeln".

(ea) Zhonghua renmin gongheguo daibu juliu tiaoli (Regeln der VR China über Verhaftung und vorläufige

Festnahme, v. 20. 12. 1954, Zhonghua renmin gongheguo fagui xuanji S. 285), \$ 2 bestimmt:

Konterrevolutionäre Elemente und andere Straftäter, die mit der Todesstrafe oder mit Freiheitsstrafe bestraft werden können, müssen nach Genehmigung durch einen Volksgerichtshof oder durch eine Volksstaatsanwaltschaft (vgl. dazu auch unten Anm. h) in Haft genommen werden. Straftäter, die in Haft genommen werden müssen, die aber eine schwere Krankheit haben oder bei denen es sich um schwangere oder ein eigenes Kind säugende Frauen handelt, kann man statt dessen gegen Bürgschaft (in Freiheit) auf die Untersuchung warten oder unter Uberwachung (in Freiheit) leben lassen.

(f) Zur Verteilung und Zuständigkeit der Gerichte sind die Angaben von Interesse, die Miyazaki Shigeki "kürzlich bei einem Besuch in China" erhalten und in Jurist 575.86 f. (vom 15. 11. 1974) wiedergegeben hat: Auf jede Provinz kommt ein Gerichtshof der Oberstufe, auf jedes Gebiet (diqu) ein Gerichtshof der Mittelstufe; ferner gibt es eine größere Anzahl von Gerichtshöfen der Grundstufe sowie Militärgerichtshöfe. (Die Stadtprovinzen Peking und Shanghai sind nicht in Gebiete unterteilt. Haragos Angaben, nach denen es in Peking nur einen Gerichtshof der Mittelstufe gibt, stehen also nicht im Widerspruch zu Miyazakis Angaben.) Alle Gerichte, auch der Oberste Gerichtshof, können in erster Instanz tätig werden. Gegen das Urteil erster Instanz ist die Berufung an das nächsthöhere Gericht möglich; dessen Urteil ist endgültig. Gewöhnliche Fälle werden von den Gerichten der Grundstufe behandelt. Die Gerichte der Mittelstufe behandeln die Fälle, in denen sie sich wegen der Bedeutung des Falles für zuständig halten; Fälle mit 
Auslandsbezug; und Fälle, die über den örtlichen Zuständigkeitsbereich eines Gerichtshofes der Grundstufe hinausgehen. Die Gerichte der Oberstufe behandeln die Fälle, in denen sie sich wegen der Bedeutung des Falles für zuständig halten, und Fälle, die sich örtlich über mehr als ein Gebiet erstrecken. Der Oberste Gerichtshof behandelt Fälle, die örtlich über den Bereich einer Provinz hinausgehen, und Fälle von schwerwiegender Bedeutung für den Staat, wie das Verfahren gegen den Täter, der in Peking einen ausländischen Diplomaten erstochen hatte. Die höheren Gerichtshöfe führen die Gerichtshöfe unter ihnen und ziehen gegebenenfalls Fälle an sich, in denen sie sich für zuständig halten.

(g) Das japanische Strafverfahren trennt sehr streng zwischen Vorverfahren und Hauptverhandlung. (Vgl. unten Anm. k). Für den japanischen Juristen ist deshalb das chinesische Vorverfahren eine besonders fremdartige Angelegenheit, und daraus resultiert wohl auch die Darstellung Asais, denn andere Berichte über das chinesische Strafverfahren haben schon früher die Rolle des Vorverfahrens ähnlich wie jetzt der Text beschrieben. Vgl. z. B. Cohen, The Criminal Process in the People's Republic of China, Cambridge (Mass.) 1968, S. $355 \mathrm{ff}$.

Eine neuere genaue Darstellung der Rolle von Vorverfahren und Hauptverfahren in China findet in dem Bericht von Hariu Seikichi, Chūgoku shin kempõka no saiban seido (Die Gerichtsordnung unter der neuen chinesischen Verfassung, Jurist 602.124). Hariu hat im Sommer 1975. China besucht. Beim Volksgerichtshof der Oberstufe in Shanghai gab man ihm auf seine Frage nact dem Ablauf von Strafuntersuchung und -verfahren folgendes hypothetische Beispiel: In einer Fabrik wird Material gestohlen. Darauf untersucht das RK der Fabrik zusammen mit den Massen der Arbeiter die Sache. Sodann erstattet man der Polizei Bericht. Wenn es nötig ist, wird der Täter festgenommen, es werden andere notwendige. Maßnahmen zur Untersuchung des Falles durchgeführt. In der Voruntersuchung wird die Haltung, wird die Einstellung des Täters genau untersucht, und er wird „erzogen“. Hierbei wie im Folgenden arbeitet die Polizei eng mit dem Parteikomitee und der Leitung des Betriebes zusammen. - Damit kann dann der Fall abgeschlossen sein. Es kann jedoch auch nötig sein, das Verfahren weiter zu verfolgen, unter Umständen bis zu einer gerichtlichen Regelung. Falls das nach der Voruntersuchung nötig scheint, findet zunächst eine „Kritik- und Kampf-Versammlung “ (doupihui) statt. Auf suchung nötig scheint, findet zunächst eine "Kritik- und Kampf-Versammlung " (doupihui) statt. Auf
dieser Versammlung legt der Täter ein öffentliches Geständnis ab. Er wird von der "Masse" seiner Kollegen

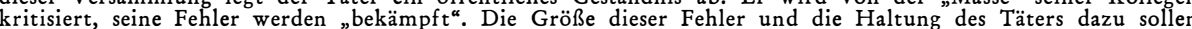
sich dabei klar zeigen. Danach sollen dann auch die Massen" - also die Kollegen - ihre Meinung dazu äußern, ob das Strafverfahren fortgesetzt werden soll. Nach der Versammlung wird der Fall mit dem Parteikomitee besprochen und ein erster Vorschlag zum Strafmaß gemacht. Auf Grund dessen kann dann eine öffentliche Verhandlung in der von Marago geschilderten Weise stattfinden. Betont wird dazu auch
hier, daß bei der Gerichtsverhandlung die Sache selbständig nochmals untersucht wird. (Zur Rolle der Staatsanwaltschaft bzw. des Staatsanwaltes der Polizei dabei vgl. unten Anm. h.) Gegen das Urteil is innerhalb von 10 Tagen Berufung an die nächste Instanz möglich. Bei Fristversäumnis ist Einlegung eines besonderen Rechtsmittels (genannt shensu, während die gewöhnliche Berufung shangsu heißt) möglich. Das Urteil der zweiten Instanz ist endgültig, nur müssen Todesurteile - wie bisher - vom Obersten Gerichtshof bestätigt werden.

Auch Hariu gegenüber wurde betont, daß man darauf achte, das Geständnis (auf das aus erzieherischen Gründen großer Wert gelegt wird, vgl. auch oben Anm. b, \$S 4, 5 der dort zitierten Regeln) als Beweis nicht überzubewerten; vielmehr müßten stets auch ausreichende andere Beweise vorliegen. Hierauf bezieht sich der zweite Satz der in einer „16-Zeichen-Direktive“ zusammengefaßten Grundsätze des Gerichtsverfahrens (im Straf- wie im Zivilprozeß):

Sich auf die Massen stützen!

(Den Sachverhalt) untersuchen!

An Ort und Stelle (den Fall) lösen!

Vor allem vermitteln! (D. h., möglichst wenig durch streitiges Urteil entscheiden.)

(h) An der juristischen Fakultät der Pekinger Universität bekam Hariu (vgl. die vorige Anm.) auf seine Fragen zur Abschaffung der Staatsanwaltschaft folgende Auskunft: Grund der Abschaffung seien die Massenlinie, die Festigung der einheitlichen Führung durch die Partei und die Vereinfachung der Verwaltung gewesen: Staatsanwaltschaft und Polizei hätten teilweise doppelte Arbeit geleistet. Deshalb diene die Abschaffung der Staatsanwaltschaft der Verwaltungsvereinfachung. Außerdem hätten sich die Staatsanwaltschaften oft als „selbständiges Königreich“ geriert, das heißt eine Art gegenseitiger Gewaltenkontrolle der "drei Justizorgane" (Polizei, Staatsanwaltschaft, Gerichte) angestrebt. Deshalb diene ihre Abschaffung der einheitlichen Führung durch die Partei und der Massenlinie. Die Abschaffung der Staatsanwaltschaft bedeute jedoch nicht, daß die besonderen staatsanwaltschaftlichen Aufgaben abgeschafft wären. Vielmehr würden diese Aufgaben nun von besonderen Beamten bei der Polizei wahrgenommen.

Nach den Universitätsjuristen gehören (wie bisher) folgende Abgaben zu den derart wahrgenommenen staatsanwaltschaftlichen Funktionen: die Ausstellung von Haftbefehlen; die Anklageerhebung; die Vertretung der Anklage in der Verhandlung; die Überwachung der Wandelung durch Arbeit (d. h. des Strafvollzugs); die Uberwachung der anderen Staatsorgane. - Wie schon Art. 97 der alten bestimmt jetzt auch Art. 27 III der neuen Verfassung, daß jeder Bürger gegen Rechts- und Pflichtverletzungen von Beamten bei "den Staatsorganen aller Stufen" Klage führen kann. Aber die gerichtliche Anklage ist in solchen Fällen "nach diesen Auskünften auch weiterhin eine staatsanwaltschaftliche Aufgabe. Ein Bürger, der einen solchen Fall vor Gericht bringen will, muß sich also an die staatsanwaltschaftliche Funktionen ausübenden Polizeibeamten wenden.

Um die von Hariu wiedergegebenen Auskünfte zusammenzufassen: die Abschaffung der Staatsanwaltschaften $\mathrm{m}$ die von Hariu wiedergegebenen Auskünfte zusall ihrer Funktionen infolge der konsequenten Durchführung der Massenlinie. Die staatsanwaltschaftlichen Funktionen werden vielmehr weiterhin als besondere Aufgaben angesehen (und in Art. 25 III der neuen Verfassung ausdrücklich erwähnt) und von besonderen Beamten ausgeübt. Nur sind diese Beamten jetzt in den Polizeiapparat eingegliedert worden, damit sie sich nicht gegenüber der Polizei oder gar gegenüber der Partei eine selbständige Rolle anmaßen. Das entspricht inoffiziellen Angaben, nach denen schon vor der Kulturrevolution mancherorts eine Verschmelzung sogar aller drei Rechtsbehörden (Gerichte, Staatsanwaltschaft, Polizei) erprobt wurde. (Cohen, Contemporary aller drei Rechtsbehörden (Gerichte, Staatsanwaltschaft, Polizei) erprobt wurde. (Cohen, Cont
Chinese Law: Research Problems und Perspectives, Cambridge (Mass.) 1970, S. 106, m. w. Nachw.).

(i) Zhonghua renmin gongheguo renminfayuan zuzhifa (Gesetz über die Organisation der Volksgerichtshöfe der VR China, v. 28. 9.1954, Zhonghua renmin gongheguo fagui xuanji S. 38), \ 7 II:

Der Angeklagte hat das Recht, sich selbst $\mathrm{zu}$ verteidigen; außerdem kann er einen Anwalt mit seiner Verteidigung beauftragen; er kann von einem von einer gesellschaftlichen Organisation empfohlen oder vom Volksgerichtshof zugelassenen Bürger verteidigt werden; er kann von nahen Verwandten oder von seinem Vormund verteidigt werden. Wenn der Volksgerichtshof es für nötig erachtet, kann er auch einen Verteidiger bestimmen, der ihn (den Angeklagten) verteidigt. “

(k) Das bezieht sich auf $\int 256$ IV der jap. StPO: „Der Anklageschrift darf kein schriftliches oder sonstiges Material beigefügt werden, beim dem befürchtet werden könnte, daß es den Richter veranlassen könnte, sich zu dem Fall bereits eine Meinung zu bilden. Auf den Inhalt derartigen Materials darf auch nicht Bezug genommen werden." (In der Entscheidung heißt es dazu, der Richter solle vor der Verhandlung ohne Meinung über den Fall, „wie ein unbeschriebenes Blatt“" sein.) 